\title{
La participation du public dans les consultations sur les projets environnementaux à grande échelle : piège ou levier de développement
}

\author{
Lucie Fréchette et Solange Van Kemenade \\ avec la collaboration de Nathalie Legrand ${ }^{1}$
}

Dans le contexte des débats actuels sur les enjeux environnementaux, les changements climatiques, le développement durable et la gouvernance, la société civile prend une place de plus en plus importante. Elle est invitée à participer à des processus de consultation des instances gouvernementales tout particulièrement dans les projets à grande échelle où l'aménagement territorial affecte les populations. Ainsi, dans le secteur de l'environnement, les impacts sociaux, économiques et culturels des projets sur les communautés ont remis à l'ordre du jour des questions relatives à la participation du public, la mobilisation collective et la consultation publique au processus démocratique sous-jacent à la prise de décision.

Par ailleurs, depuis une trentaine d'années, on assiste à une prise de conscience sur la nécessité d'inclure la dimension sociale dans les évaluations environnementales. La place de la société civile dans le processus d'évaluation environnementale et la prise en compte des effets socio-économiques et culturels que les projets pourraient entrainer pour les populations locales ont été des questions d'actualité au Québec entre autres autour des projets des ports méthaniers de Cacouna et Rabaska et dans le débat sur la privatisation d'une partie du Parc du MontOrford. On constate dans ces cas, que le débat autour d'un projet peut mener à des mobilisations populaires élargies qui visent à influencer les processus décisionnels.

Même si le discours sociopolitique courant considère la communauté, au sens des collectivités locales ou régionales et au sens des groupes d'intérêt, comme un public à privilégier, voire un partenaire, on constate que la définition de la participation significative du public n'est pas la même pour tous les acteurs ${ }^{2}$. Il s' agit là du point de départ de cet article issu d'une recherche menée dans le cadre de l'Alliance de recherche universitécommunauté en innovation sociale et développement des communautés (ARUCISDC $)^{3}$. Il veut clarifier ce qu'on entend par participation significative du public et analyser dans quelles conditions cette participation devient significative. Finalement, nous terminerons en examinant comment la participation se transforme en piège neutralisant la mobilisation des communautés ou en levier de leur développement.

\section{Qu'entend-on par participation significative?}

Le concept de «participation significative » ainsi que des concepts associés tels que «large soutien communautaire» (Broad community support) et «acceptabilité sociale» (Social acceptability) ou encore le concept de Social licence plus employé dans le secteur minier canadien sont apparus récemment en guise de reconnaissance du 
droit qu'ont les populations de se prononcer sur les aménagements territoriaux ou sur les projets à grande échelle pouvant les affecter. La Loi canadienne sur l'évaluation environnementale fournit un cadre législatif pour la tenue des évaluations environnementales des projets entraînant une décision réglementaire du gouvernement. L'intensité de l'implication $\mathrm{du}$ public peut varier selon le type d'évaluation environnementale requise, soit un examen préalable, une étude approfondie, ou une commission d'examen. Toutefois, bien que la loi soit précise quant à la définition du public, elle l'est beaucoup moins en ce qui a trait à une définition claire et complète du concept de participation significative en tant que telle. Elle est tout aussi floue en ce qui a trait à des exigences susceptibles d'estimer, de façon adéquate, qu'une participation significative soit bien atteinte. Divers documents tirés de la littérature gouvernementale canadienne distinguent des niveaux de sollicitation du public. Il en émerge trois: 1- l'information qui constitue une communication à sens unique, 2- la consultation qui constitue une communication à double sens avec échanges d'informations, 3- la participation significative qui constitue une communication à sens multiple où les diverses parties collaborent à la conception d'un processus et apprennent des autres. En fait, il ne suffit pas d'entendre les avis et d'écouter les populations, il apparaît nécessaire d'aller plus loin en donnant la possibilité au public d'avoir une influence sur les décisions le concernant $^{4}$, d'où l'apparition du concept de «participation significative du public ».

Une large recension sur le sens de la participation dans des écrits gouvernementaux, ceux d'ONG et dans la littérature scientifique et la consultation de plusieurs sites internet ont permis d'identifier dans les définitions retenues un tronc commun où participation et consultation sont souvent interchangeables et envisagées comme des concepts dynamiques ou des processus comme on le voit dans les exemples qui suivent. Les définitions et surtout les textes les détaillant bifurquent ensuite en fonction des champs d'intérêt des organisations.

o «Processus interactif et itératif ayant pour but d'obtenir les points de vue des gens, de les prendre en considération et de fournir à ces derniers l'occasion d'influer sur les décisions.» Environnement Canada.

o "Relation basée sur un partenariat avec les administrations, dans lesquelles les citoyens sont activement engagés dans les processus décisionnels et d'élaboration des politiques. Cette relation reconnaît aux citoyens la possibilité de proposer des options et d'orienter le dialogue sur les politiques - même si la responsabilité de la décision finale ou de la formulation des politiques continue d'incomber au gouvernement. " Organisation de coopération et de développement économique (OCDE).

- «Signifie que les citoyens ont la possibilité de partager la prise de décision en exprimant leurs points de vue et leurs préférences quant aux politiques de santé et en faisant des choix individuels et collectifs, de ce fait en étant un partenaire actif du processus de décision.» Organisation mondiale de la santé (OMS).

○ «Processus par lequel les parties prenantes influencent les initiatives de développement, les décisions et les ressources qui les concernent, sur lesquelles elles ont un droit de regard. " Groupe d'étude sur le développement participatif de la Banque mondiale.

En combinant les éléments composant une quinzaine de définitions, nous dégageons notre définition du processus de participation significative. Cette formulation large en trois volets précise la nature du public impliqué, le caractère récurrent de la participation, le pouvoir d'influence du public et la rétroaction attendue de la part des décideurs. 
Processus interactif parfois récurrent impliquant des individus ou des groupes qui sont affectés ou intéressés par un projet et qui font valoir leurs avis, proposent des options ou orientent les débats. Processus qui offre l'occasion d'exercer une influence sur le contenu d'un projet avant la prise de décision finale par ses promoteurs ou par les autorités qui en sont responsables. Processus invitant les promoteurs ou responsables de projets à rendre compte de l'influence de la participation du public sur leurs choix ou décisions.

La littérature consultée permet aussi de dégager des exigences qualifiant le processus de consultation pour que l'on puisse en arriver à une participation significative du public. Nous les résumons au tableau 1 en indiquant les noms des organisations et ministères y référant.

Tableau 1 : Facteurs favorisant les processus consultatifs avec participation significative du public

\begin{tabular}{|c|c|c|}
\hline Exigences & Signification & Instances y référant entre 1996 et 2006 \\
\hline Participation précoce & $\begin{array}{l}\text { Le public doit être impliqué } \\
\text { suffisamment tôt pour que ses } \\
\text { recommandations ou avis soient pris en } \\
\text { compte avant que les choix décisifs ne } \\
\text { soient déjà faits et pendant que les } \\
\text { options sont encore débattues; } \\
\text { Le public doit être impliqué dès les } \\
\text { premières étapes (dès la conception) de } \\
\text { du projet. }\end{array}$ & $\begin{array}{l}\text { Environnement Canada ; Santé Canada } \\
\text { Agence canadienne d'évaluation } \\
\text { environnementale (ACÉE) ; Banque } \\
\text { mondiale; Organisation mondiale de la santé } \\
\text { (OMS) ; Organisation de coopération et de } \\
\text { développement économique (OCDE); } \\
\text { Commission mixte internationale. }\end{array}$ \\
\hline Transparence & $\begin{array}{l}\text { Les objectifs visés par la participation } \\
\text { doivent être définis et clairement } \\
\text { présentés au public; } \\
\text { Les étapes du processus doivent être } \\
\text { clairement expliquées; } \\
\text { Aucune information ne doit être cachée } \\
\text { volontairement au public; } \\
\text { Le public doit être averti d'emblée des } \\
\text { limites associées à sa participation; } \\
\text { Le calendrier des décisions doit être } \\
\text { annoncé au début puis respecté. }\end{array}$ & $\begin{array}{l}\text { Banque mondiale; Association internationale } \\
\text { pour l'évaluation d'impacts (IAIA); OMS ; } \\
\text { Environnement Canada; Canadian School of } \\
\text { Public Service; Diversification de } \\
\text { l'Économie de l'Ouest Canada (DEO); } \\
\text { OCDE Santé Canada ; Banque } \\
\text { Interaméricaine de Dévelopment (BID); Loi } \\
\text { canadienne sur l'évaluation } \\
\text { environnementale (LCÉE). }\end{array}$ \\
\hline Partage d'information & $\begin{array}{l}\text { Les promoteurs des projets doivent } \\
\text { fournir de l'information fiable, } \\
\text { pertinente, objective et compréhensible } \\
\text { pour les diverses parties; } \\
\text { L'information doit être transmise via des } \\
\text { moyens technologiques adaptés aux } \\
\text { contextes; } \\
\text { Doit être transmise en temps opportun de } \\
\text { façon à ce que le public puisse l'utiliser } \\
\text { dans sa réflexion et sa préparation; } \\
\text { Doit être transmise dans un langage } \\
\text { compréhensible par tous; } \\
\text { Doit être accessible et gratuite pour tous. }\end{array}$ & $\begin{array}{l}\text { Banque mondiale; Association canadienne } \\
\text { des normes (ACNOR); Commission } \\
\text { économique Nations Unies pour l'Europe } \\
\text { (CÉE); Association internationale pour la } \\
\text { participation du public (AIPP); IAIA ; OMS; } \\
\text { Environnement Canada ; DEO ; OCDE; } \\
\text { Santé Canada; Directive - LCÉE ; ACÉE; } \\
\text { Fédération française des associations de } \\
\text { protection de la nature et l'environnement. }\end{array}$ \\
\hline
\end{tabular}




\begin{tabular}{|c|c|c|}
\hline Équité & $\begin{array}{l}\text { Le processus de consultation doit être } \\
\text { accessible à tous les acteurs } \\
\text { préalablement désignés; } \\
\text { Accès équitable à une aide financière; } \\
\text { Le modérateur ne doit avoir aucun parti } \\
\text { pris quant au déroulement et aux résultats } \\
\text { attendus de la consultation; } \\
\text { Les consultations doivent avoir lieu dans } \\
\text { des endroits qui ne favorisent aucune des } \\
\text { parties impliquées. }\end{array}$ & $\begin{array}{l}\text { CÉE; Banque mondiale ; AIPP; IAIA; OMS; } \\
\text { OCDE; Santé Canada; ACÉE BID; Directive } \\
\text { ministérielle - LCÉE. }\end{array}$ \\
\hline $\begin{array}{l}\text { Obligation de rendre } \\
\text { compte de l'influence des } \\
\text { avis du public sur la } \\
\text { décision }\end{array}$ & $\begin{array}{l}\text { Des mécanismes doivent être mis sur pied } \\
\text { afin de garantir au public que ses avis ont } \\
\text { été pris en compte dans la décision; } \\
\text { Des rétroactions doivent être effectuées } \\
\text { régulièrement au public sur l'influence } \\
\text { qu'il a sur les diverses décisions. }\end{array}$ & $\begin{array}{l}\text { CÉE; AIPP; OMS; OCDE; Environnement } \\
\text { Canada ; IAIA;DEO;BID; Canadian School } \\
\text { of Public Service; Directive ministérielle- } \\
\text { LCÉE. }\end{array}$ \\
\hline Respect & $\begin{array}{l}\text { Les avis ainsi que les individus ou } \\
\text { organisations les présentant doivent être } \\
\text { accueillis avec respect et ouverture; } \\
\text { Doit tenir compte des préoccupations du } \\
\text { public; } \\
\text { Respect des valeurs et des besoins du } \\
\text { public; } \\
\text { Un code de bonne conduite peut être } \\
\text { instauré } \\
\text { Les volets social, environnemental et } \\
\text { économique doivent être considérés }\end{array}$ & $\begin{array}{l}\text { IAIA ; Centre de recherche en } \\
\text { développement international (CRDI); } \\
\text { Canadian School of Public Service; DEO; } \\
\text { Santé Canada; Directive ministérielle - } \\
\text { LCÉE ; OCDE; ACÉE }\end{array}$ \\
\hline Flexibilité/Souplesse & $\begin{array}{l}\text { La participation du public doit être } \\
\text { adaptée aux contextes culturel, social, } \\
\text { politique particuliers dans lesquels elle } \\
\text { s'inscrit et adaptée aux objectifs } \\
\text { poursuivis; } \\
\text { Les méthodes de participation, les } \\
\text { processus, l'information, etc. doivent être } \\
\text { adaptés selon les contextes; } \\
\text { Il n'y a pas de recette magique ou } \\
\text { universelle qui puisse être appliquée à } \\
\text { l'ensemble des consultations. }\end{array}$ & $\begin{array}{l}\text { IAIA ; OMS ; Environnement Canada ; } \\
\text { OCDE; Santé Canada ; BID; Directive } \\
\text { ministérielle -LCÉE; ACÉE. }\end{array}$ \\
\hline
\end{tabular}

\section{Qu'attend-on de la participation significative du public?}

Il appert que le processus de participation $\mathrm{du}$ public constitue non pas un but en soi mais bien un moyen, un outil pour atteindre des objectifs préalablement définis ${ }^{5}$. Il est donc important d'identifier les raisons pour lesquelles les initiateurs des consultations souhaitent faire
Il appert que le processus de participation du public constitue non pas un but en soi mais bien un moyen, un outil pour atteindre des objectifs préalablement définis. participer de façon significative le public. Les rapports et directives des organisations gouvernementales canadiennes ainsi que ceux des organisations internationales révèlent que l'on s'attend à ce que la consultation du public permette de répondre aux besoins et aux intérêts locaux, 
conduise à la réalisation d'un développement et de solutions durables, conduise à une réception des décisions plus favorable par le public, augmente la crédibilité du processus, augmente la légitimité des décisions, réduise les conflits et conduise à une bonne gouvernance.

Des attentes comme celles-là exigent l'octroi de ressources humaines et financières aux opérations de consultation et en soutien à la participation. Elles exigent aussi qu'on accorde du temps aux processus consultatifs. Finalement, les responsables des processus de consultation ne peuvent escompter de tels résultats sans prendre en compte la rétroaction de la population. Une analyse de quatre projets de développement de ressources naturelles au Canada, dont un est encore soumis au processus d'évaluation environnementale, indique que passer de la bonne intention à la réalisation optimale n'est pas un défi anodin.

Les quatre projets sont : Énergie Cacouna et Rabaska, deux ports avec des terminaux méthaniers au Québec, le projet d'hydroénergie Eastmain 1-A également au Québec, et gazoduc Mackenzie. Ce dernier localisé dans les territoires du Nord-Ouest traversera plusieurs provinces canadiennes. Nous avons examiné avec un intérêt particulier comment les instances de consultation s'étaient déroulées dans chacun de cas. Nous avons comparé avec des projets similaires et cela même au niveau international, dans des pays où ce type des projets avait gagné une certaine acceptabilité sociale.

Dans les cas de Cacouna et de Rabaska, les projets ont suscité des vives réactions à cause de l'emplacement qui était considéré peu sécuritaire. La population a estimé également que des valeurs telles que la qualité de vie, les aspects visuels, le patrimoine historique de la communauté et leur sécurité seraient compromises par le développement de tels projets. Bien que les promoteurs aient mené des processus comprenant la diffusion des informations relatives aux projets incluant un sondage d'opinion publique dans le cas de Rabaska, ces processus n'ont pas été considérés satisfaisants par les communautés fort partagées quant à la question et le projet, malgré l'approbation gouvernementale accordée continue à être l'objet d'un grand débat public. Dans le cas de Cacouna, un referendum populaire a été organisé par la communauté dont les avis étaient aussi divisés sur la question. Quant au gazoduc Mackenzie, le processus d'évaluation environnementale a été suspendu en novembre 2006, par une décision de la Cour fédérale en faveur de l'une des Premières Nations impliquées dans le processus. Cette population estimait ne pas avoir été consultée de manière appropriée. Finalement, les communautés cries impliquées dans le processus d'évaluation environnementale d'Eastmain-1-A, récemment approuvé par les instances gouvernementales, ont manifesté leur opposition au projet en raison de ces impacts potentiels et pour ne pas avoir été suffisamment consultées. Elles ont manifesté également manquer d'information sur le projet. D'autres témoignages faisaient ressortir l'importance d'avoir de l'information dans la langue autochtone.

En somme, ces projets partagent les caractéristiques suivantes :

- Il s'agit dans tous les cas des projets controversés;

- Les projets ne jouissent pas «d'acceptabilité sociale »;

- Dans tous les cas, des processus de consultation de la population ont été conduits en forme parallèle ou à posteriori de ceux organisés par les promoteurs;

- Ces processus ont été initiés parce que les mécanismes de consultation «officiels» ont été jugés inappropriés ou insatisfaisants par la population; 
- Ces consultations ont eu comme effet de diviser la population locale affectant la cohésion sociale de la communauté. .
- La voix des peuples autochtones n'a pas été suffisamment entendue lors des consultations.

\section{La participation : un concept teinté de rapports de pouvoir}

Les définitions et composantes précédentes de la participation dans les processus de consultation ne peuvent être interprétées qu'à la lumière des contextes sociaux, culturels et politiques associés à leur déploiement. Le réflexe de consultation et plus encore l'ouverture à la participation de la population dans l'évaluation prospective des grands projets de société, projets environnementaux ou autres, n'est pas un automatisme chez les pouvoirs publics. L'histoire des mouvements sociaux en fait foi. La participation des populations a été durement gagnée au cours des siècles.

Même si dans une société démocratique la participation ou encore la citoyenneté sont partie prenante du discours et des mœurs, les mouvements sociaux et l'organisation communautaire demeurent vigilants pour activer la voix et le pouvoir d'agir des citoyens. Les enjeux environnementaux liés aux grands projets de développement n'échappent pas à cette vigilance. En effet, les dernières décennies ont vu émerger un fort mouvement qualifié d'écologique ou d'environnemental. Les jeunes générations y adhèrent avec beaucoup de conviction tout comme les militants engagés dans la lutte contre les iniquités sociales dans l'aménagement des territoires. La citoyenneté $\mathrm{y}$ trouve un terrain d'application non négligeable dont certains parlent en termes d'écocitoyenneté ${ }^{67}$.

La réaction des acteurs aux projets à composantes environnementales prend des formes des plus variées depuis la contestation sous forme de lobby, de participation à des consultations ou des consultations parallèles, de revendications collectives jusqu'à la désobéissance civile. L'action se fait parfois locale, parfois régionale et parfois à large échelle tant nationale que transcontinentale. Peu importe l'échelle où le citoyen ou des groupes sont convoqués à débattre de projets d'aménagement, on a alors affaire à un exercice de débat public qui pose les citoyens en acteurs engagés dans le processus de planification même dans un cadre où les décisions finales se prennent en dehors de l'enceinte des délibérations. La participation à un processus de consultation ou à une délibération implique forcément que les décideurs acceptent de partager au moins une partie du pouvoir avec les populations.

\section{La participation à un processus de consultation ou à une délibération implique forcément que les décideurs acceptent de partager au moins une partie du pouvoir avec les populations.}

Comme le mentionne $\mathrm{Subra}^{8}$ «Le débat public n'est pas seulement un moment où s'affrontent et s'échangent des arguments. C'est aussi un moment où se constitue du pouvoir et où les rapports de forces et les relations entre acteurs se modifient ». Dans les projets de développement à grande échelle, on pourrait donc se demander si les dispositifs publics favorisent vraiment un partage du pouvoir ou une influence dans la prise de décisions ou si la mise en place d'un processus d'élargissement de l'information et de consultation auprès de citoyens, ne constitue pas plutôt une stratégie de réduction des tensions et d'amélioration de la concertation entre des groupes entretenant des intérêts divergents. Il paraît éloquent que le 
débat sur des projets de développement énergétique récemment annoncé au Canada, a conduit dans tous les cas à la tenue des référendums populaires ou à des processus de consultation indépendants de ceux organisés par les développeurs auprès des populations potentiellement affectées.

Si la participation du public s'inscrit dans un contexte politisé où l'annonce d'un grand projet est le point de mire des ministères ou du gouvernement, la consultation est souvent piégée. En effet, un projet annoncé se transforme souvent en projet à défendre. La consultation s'inscrit alors dans une formule Décider-Annoncer- Informer-Défendre. Si l'annonce est de l'ordre de l'intention d'ouvrir un grand chantier sans que la décision quant à la façon dont il se développera soit prise, la participation a plus de chance de se traduire par la formule Proposer- informer -Consulter- ÉtudierDécider. La participation du public pourrait alors s'avérer significative.

\section{Se mettre le pied dans un piège ou délibérer collectivement}

$\mathrm{Si}$ nous effectuons une relecture des retombées à la lumière de leçons tirées d'expériences de consultation, on peut se demander si l'invitation à la participation du public n'est pas qu'un piège neutralisant la mobilisation populaire ou une invitation à délibérer collectivement en vue d'un développement optimal. On peut aussi en dégager l'idée que l'évolution de la participation du public dans des conditions favorables s'avère une avancée démocratique et un levier en faveur du développement social et économique. Piège ou levier ? la réponse est tributaire des conditions dans lesquelles se conçoit la consultation et s'exerce la participation.

La réponse aux besoins et intérêts locaux implique une façon de gérer la participation qui donne la parole aux citoyens. Certaines consultations se limitent à une liste pré-établie de consultés ce qui induit des choix dont la neutralité est questionnable. La consultation institutionnalisée donne une tribune aux opposants aux projets qui sont souvent les premiers à se mobiliser pour prendre la parole publiquement. La tentation est forte chez les promoteurs de chercher à assourdir leur point de vue.

Un des effets escomptés par les promoteurs des projets est de rendre les grands projets désirables ou du moins acceptables par les populations. L'intention est louable si elle s'appuie sur des processus d'information de qualité. On a ici affaire à un des ingrédients essentiels fondant le débat public. L'information doit alors être envisagée tout autant dans sa forme ascendante (des participants vers les promoteurs ou les ministères concernés) que descendante (des experts, des promoteurs et des ministères vers les populations, groupes ou citoyens). Il importe alors de faire une évaluation éclairée de ce qui constitue une information de qualité (quantité d'information, pertinence des données, données essentielles, degrés de vulgarisation nécessaire et acceptable, stratégies, budgets, etc.). Dans le cas des projets qui affectent les peuples autochtones, cette information devrait être disponible dans la langue de ces communautés et les rencontres d'information compter des dispositifs qui assureront la bonne compréhension du projet et de ses enjeux.

Mentionnons enfin que le volume d'information disponible peut être considérable, voire démesuré et les populations sont ainsi confrontées à la difficulté de gérer cette surabondance d'information. En effet, il arrive souvent que les organisations locales disposent de dossiers 
bien étoffés sur diverses questions associées aux grands enjeux de l'heure comme on en trouve sur l'eau, les transports, les énergies non renouvelables, les écosystèmes, les ressources naturelles et bien d'autres. Filtrer et retenir de l'information tout comme l'excès d'information sont des pièges à éviter ou au mieux des défis difficiles à gérer dans le contexte de la participation des communautés dans le cadre de processus de consultation.

On cite la précocité de l'action comme exigence de la participation tout comme on mentionne aussi la flexibilité pour caractériser l'action de consultation au long de son processus. Bien sûr, l'on comprendra rapidement que la consultation n'a de sens que si le projet n'est pas encore ficelé. D'où l'exigence de la précocité de l'action. Elle n'a cependant de sens que si l'ouverture à l'influence des participants est réelle. Le débat public au-delà de l'information a pour originalité de recevoir des contre-projets ou des suggestions de modifications à la lumière de la contre-expertise ou d'éclairages complémentaires des communautés ${ }^{9}$.

La consultation qui permet le débat est ouverte aux savoirs de natures diverses, savoir des experts, des techniciens, des groupes de pression ou d'intérêts, des communautés locales, des citoyens. Le débat revêt ainsi un caractère évolutif dans le temps. Le point de vue a le temps de se forger et les positions se nuancent à la lumière des choix envisageables. C'est dans ces conditions qu'on pourra parler de retombées en termes de décisions plus éclairées.

\section{En guise de conclusion : participation significative, gouvernance, et démocratie}

La participation significative des citoyens, des mouvements sociaux, des groupes d'intérêt, fait partie intégrale de la bonne gouvernance. Elle contribue sans doute à l'exercice et au maintien de la démocratie. Le lien entre la participation significative et la bonne gouvernance est associé aux pratiques démocratiques à des échelles variables, locales, régionales ou nationales. Elle est aussi associée à un ou des projets de société empreints de justice sociale qui prend ici forme de justice environnementale. Les mouvements sociaux concernés par les questions environnementales font leur ce principe de justice environnementale tout comme l'insèrent de plus en plus dans leur cadre de travail des organisateurs communautaires du secteur de la santé et des services sociaux, du moins au Québec.

Les populations touchées par les projets de développement à grande échelle bénéficient de l'apport de l'organisation communautaire en raison de son savoir-faire dans la mobilisation des communautés, la concertation et le partenariat. Un savoir-faire qui outille les citoyens et les groupes pour mieux prendre la parole, évaluer leur marge de manœuvre dans les luttes de pouvoir, pour développer des argumentaires en lien avec les propositions des pouvoirs publics ou de l'entreprise privée et développer des actions en faveur de la transformation harmonieuse des milieux de vie et des territoires.

La bonne gouvernance est ainsi aussi affaire d'équilibre entre les pouvoirs et contrepouvoirs. La mise en place d'instances de participation n'est pas garante du renforcement de la démocratie. Elle crée au moins l'occasion de provoquer un questionnement sinon une redéfinition des relations entre l'État, ses institutions, les élus, les groupes et les citoyens. Bhérer ${ }^{10}$ souligne que la participation institutionnalisée est aux confins de deux tendances; l'élargissement de l'espace démocratique et le danger de la récupération politique des citoyens organisés. 
$\mathrm{Au}$ terme de cette réflexion, nous constatons que la participation des communautés dans le cadre des processus de consultation sur des projets à grande échelle, en dépit des pièges qui la guettent, est un acquis à préserver avec vigilance et à soumettre constamment à l'analyse critique. C'est la condition pour qu'elle actualise son potentiel de démocratisation de la décision en matière de développement environnemental et de développement des communautés.

\section{Notes et références}

1 Lucie Fréchette, docteure en psychologie est professeure au Département de travail social et des sciences sociales de l'Université du Québec en Outaouais et dirige l'Alliance de recherche université communauté en innovation sociale et développement des communautés (ARUC-ISDC). Solange Van Kemenade, docteure en sociologie, est chercheure à l'Agence de la santé publique du Canada et membre de l'ARUC-ISDC. Nathalie Legrand, maîtrise en relations industrielles, est professionnelle de recherche.

2 Dalal-Clayton B. \& S. Bass (comp.) (2002). Sustainable Development Strategies - A Resource Book, The International Institute for Environment and Development (IIED), Organisation for Economic Co-operation and Development, Paris and United Nations Development Programme, New York, 358 p.

3 Cette recherche a été publiée par l'ARUC-ISDC sous la forme de Cahier de recherche disponible à l'adresse http://www.uqo.ca/observer/DevLocal/Gouvernance/Consultation.htm

4 Danforth, V. (2006). "La diffusion et le partage de l'information sont indispensables pour assurer un développement durable» dans La Lettre d'information trimestrielle du Groupe de la Banque Mondiale au Maghreb, N0. 2, 70-71.

5 OCDE (2002). Des citoyens partenaires - Information, consultation et participation à la formulation des politiques publiques, et - Manuel de l'OCDE sur l'information, la consultation et la participation à la formulation des politiques publiques, www.oecd.org Consulté le 19 mars 2007.

6 Tremblay, P.-A.(2005) «Du pas dans ma cour à l'écocitoyenneté. Opposition aux centrales hydroélectriques privées et projets locaux de société », Nouvelles pratiques sociales, vol. 18 no 1, p.26-38.

7 Maldonado-Gonzalez, A-L. et M.Cook (2007) «Organisation communautaire et environnement», dans L'organisation communautaire. Fondements, approches et champs de pratique sous la dir. de D. Bourque, Y. Comeau, L. Favreau et L. Fréchette, Presses de l'Université du Québec, Québec,p. 181-190.

8 Subra, P. (2003) A quoi et à qui sert le débat public ? dans Hérodote. Revue de géographie et de géopolitique, no 110, p.149-170

9 Fourniau, J.M. (2001) «L'expérience du débat public institutionnalisé : vers une procédure démocratique de décision en matière d'aménagement ?, dans Annales des mines, octobre, p. 67-80.

10 Bherer, L. (2006) «La démocratie participative et la qualification citoyenne à la frontière de la société civile et de l’État », Nouvelles pratiques sociales, vol 18no2,p.2438. 


\section{Pub}

\section{Les études de cycles supérieurs à l'UQAC}

